\title{
Adjusting Na doping via wet-chemical synthesis to enhance thermoelectric properties of polycrystalline SnS
}

\author{
Huaichao Tang ${ }^{1}$, Jin-Feng Dong ${ }^{1}$, Fu-Hua Sun ${ }^{1}$, Asfandiyar ${ }^{1}$, Pengpeng Shang ${ }^{2}$ and Jing-Feng Li ${ }^{{ }^{*}}$
}

ABSTRACT Tin sulfide ( $\mathrm{SnS}$ ) has analogous structural features to tin selenide ( $\mathrm{SnSe}$ ), but contains more abundant resources as compared with $\mathrm{SnSe}$. SnS has elicited attention as a potential eco-friendly thermoelectric (TE) material. However, the intrinsic carrier concentration of $\mathrm{SnS}$ is very low, thereby hindering the performance improvement of the material. This study proposes that the TE properties of polycrystalline $\mathrm{Na}$ doped SnS (synthesized through an improved chemical coprecipitation) can be significantly enhanced. The maximum power factor (PF) of $362 \mu \mathrm{W} \mathrm{m} \mathrm{K}^{-1}$ at $873 \mathrm{~K}$ was achieved, presenting a state-of-the-art value for the polycrystalline $\mathrm{SnS}$. Considering the merits of the improved electrical properties and lower thermal conductivity of $S n S$, the highest $Z T$ was up to 0.52 at $873 \mathrm{~K}$ even without intentional chemical doping. This study offers an effective approach for improving the PF to achieve high $Z T$ in SnS. Hence, we expect that this new perspective can be extended to other dopants and broaden the scope of synthesis technology.

Keywords: SnS, rapid preparation, Na doping, thermoelectric

\section{INTRODUCTION}

Thermoelectric (TE) materials, which can directly and reversibly convert heat into electricity, have attracted increasing attention both in scientific and industrial fields [1-3]. The dimensionless figure of merit $Z T=\left(\sigma \alpha^{2} / \kappa\right) T$ is a key indicator that determines the TE conversion efficiency [4-6], where $\sigma, \alpha, \kappa$ and $T$ are the electrical conductivity, Seebeck coefficient, thermal conductivity, and working temperature in Kelvin, respectively. Good TE materials possess not only high $\sigma$ and large $\alpha$, but also low thermal conductivity [7-9]. However, the highly coupled relationship among these parameters makes it difficult to improve the $Z T$ value [10-12]. The concept of "phonon-glass electron crystal" proposed by Slack in the 1990s [13-15] has inspired people to identify the unique structures of TE materials that transfer carriers smoothly, while blocking phonon transmission, and explore new strategies, such as band engineering [16,17] and manipulation of phonon transport by various defects $[18,19]$. These explorations have been applied to the materials, such as $(\mathrm{Bi}, \mathrm{Sb})_{2} \mathrm{Te}_{3}$ [20,21], PbTe [22,23], $\mathrm{Ag}_{9} \mathrm{GaSe}_{6}$ [24], BiCuSeO oxyselenides [25], chalcogenides and selenides [3,26-32].

Recently, a simple binary compound SnS [33,34], with low-cost and social acceptance advantages, has emerged as a potential high-performance TE material. The SnS, with analogous structural features of SnSe [35], belongs to the orthorhombic (Pbmn) space group [36], as shown in Fig. 1. The connection among layers belongs to weak van der Waals force, thus, leading to low thermal conductivity [37]. As a nonstoichiometric compound, SnS naturally presents the intrinsic p-type semiconductor [38]. Li et al. [34] first reported the TE performance of pure SnS. Thereafter, the doped SnS with other elements, such as $\mathrm{Ag}$ and $\mathrm{Na}$, has been successfully investigated and the $Z T$ value increased up to $0.6[29,33]$. Recently, a single crystal of SnS, particularly, for the example of $\mathrm{Na}$ doping, has been successfully used to improve the TE performance. Zhou et al. [26] and Zhao et al. [28] reported that $Z T$ values of Na-doped SnS single crystals exceeded 1.0 at $\sim 870 \mathrm{~K}$. Nevertheless, the addition of a valuable metal, $\mathrm{Ag}$, and an inflammable, explosive metal, $\mathrm{Na}$, may minimize the practical application of $\mathrm{SnS}$ to a large extent. On the other hand, the poor mechanical properties and complex preparation processes of $\mathrm{SnS}$

\footnotetext{
${ }^{1}$ State Key Laboratory of New Ceramics and Fine Processing, School of Materials Science and Engineering, Tsinghua University, Beijing 100084, China

${ }^{2}$ College of Chemistry and Materials Science, Shandong Agricultural University, Taian 271018, China

* Corresponding author (email: jingfeng@mail.tsinghua.edu.cn)
} 


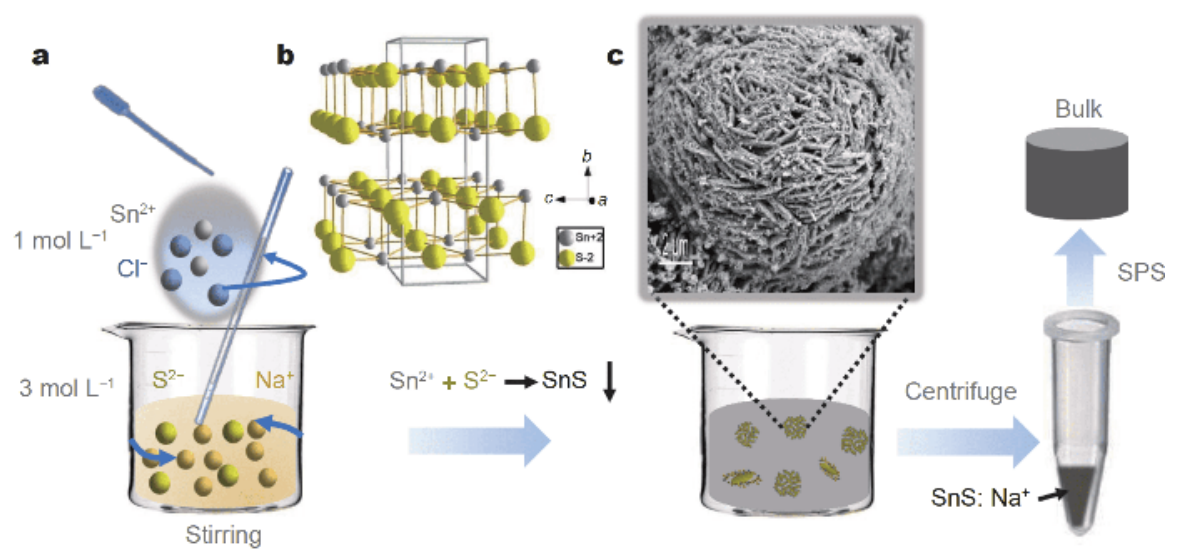

Figure 1 (a) Schematic of the experimental setup used for synthesizing polycrystalline $\mathrm{Sn}_{1-x} \mathrm{Na}_{x} \mathrm{~S}$ nanoparticles and bulk. (b) Crystal structure of SnS in the Pnma phase at room temperature. Color designations are as follows: Sn (gray) and S (yellow). (c) Field emission scanning electron microscopy (FESEM) image of the SnS: $\mathrm{Na}^{+}$powder.

single crystals can limit their industrial production. Moreover, owing to the strong oxidation of $\mathrm{Sn}^{2+}$, preparing pure $\mathrm{SnS}$ without $\mathrm{Sn}^{4+}$ (e.g., $\mathrm{SnO}_{2}$ and $\mathrm{SnS}_{2}$ ) [39] becomes a considerable issue.

Based on the above consideration, we attempted to dope $\mathrm{Na}$ into polycrystalline $\mathrm{SnS}$ using an improved chemical co-precipitation technology to eliminate and/or avoid the above mentioned problems. The schematic of the experimental setup is presented in Fig. 1a. Interestingly, the content of doped $\mathrm{Na}$ ions in SnS bulks can be determined by altering the filtration conditions. The result shows that the conductivity of SnS increased by even two orders of magnitude after introducing $\mathrm{Na}$ ions, especifically at low temperatures. Accordingly, the power factor $\left(\mathrm{PF}=\sigma \alpha^{2}\right)$ of Na-doped $\mathrm{SnS}$ increases up to $362 \mu \mathrm{W} \mathrm{m}{ }^{-1} \mathrm{~K}^{-2}$ at $873 \mathrm{~K}$, which is presently the highest reported value for the polycrystalline SnS. Therefore, the maximum $Z T$ value is 0.52 at $873 \mathrm{~K}$ in the sample centrifuged for three times. Our results suggest that this preparation technique can be extended to other dopants and compounds.

\section{EXPERIMENTAL SECTION}

\section{Synthesis}

In this experiment, all the raw materials, including $\mathrm{SnCl}_{2} \cdot 2 \mathrm{H}_{2} \mathrm{O}, \mathrm{Na}_{2} \mathrm{~S} \cdot 9 \mathrm{H}_{2} \mathrm{O}$, hydrochloric acid, anhydrous alcohol, glycol, and hydrazine hydrate, were used as analytical grade without further purification. The $1 \mathrm{~mol} \mathrm{~L}^{-1} \mathrm{SnCl}_{2} \cdot 2 \mathrm{H}_{2} \mathrm{O}$ solution was added dropwise into the mixture of $3 \mathrm{~mol} \mathrm{~L}^{-1} \mathrm{Na}_{2} \mathrm{~S}$ solution and trace amount of hydrazine hydrate. Hereafter, the solutions were centrifuged and washed two, three, and four times and were named as Centrifuged-2c, Centrifuged-3c, and Centrifuged-4c, respectively. The precipitates were dried in a vacuum-drying oven for $24 \mathrm{~h}$ prior to further use. Then, the powders were loaded into a $\Phi 12$ graphite die in air and sintered into bulks by spark plasma sintering (SPS-211Lx, Fuji Electronic Industrial) at $933 \mathrm{~K}$ for $5 \mathrm{~min}$ under a pressure of $50 \mathrm{MPa}$.

\section{Material characterization}

The phase, morphology, and composition of the samples were characterized via X-ray diffraction (XRD) with $\mathrm{Cu}$ Ka radiation (XRD, D8 Advance; Bruker, Germany, $\lambda=$ 1.5418 Å), FESEM (Merlin VP Compact Zeiss, Germany), and transmission electron microscopy (TEM, 2100F; JEOL, Japan), respectively. The electrical transport property, $\sigma$ and $\alpha$ were measured using Ulvac-Riko ZEM-2. The density $\rho$ was measured using the Archimedes method. Compared with the theoretical density, $\rho$ of all the samples is more than $90 \%$ individually for each sample. The specific heat capacity, $C p$, was estimated from the experimental data, as shown in Fig. S1A (Supplementary Information). The thermal diffusivity, $D$, (Fig. S1B) was simultaneously measured using the laser ash method (TC9000; Ulvac-Riko, Japan). Then, the thermal conductivity, $\kappa$, was calculated according to the function $\kappa=D C \mathrm{p} \rho$. The measurement uncertainty in the $Z T$ value is approximately $20 \%$, which is caused by $\alpha, \sigma, \kappa, D$, and $\rho$ within $3 \%-5 \%$.

\section{RESULTS AND DISCUSSION}

The crystal lattice of SnS, with analogous structural features of SnSe, belongs to the orthorhombic (Pbmn space) group at room temperature, as shown in Fig. $1 \mathrm{~b}$. 

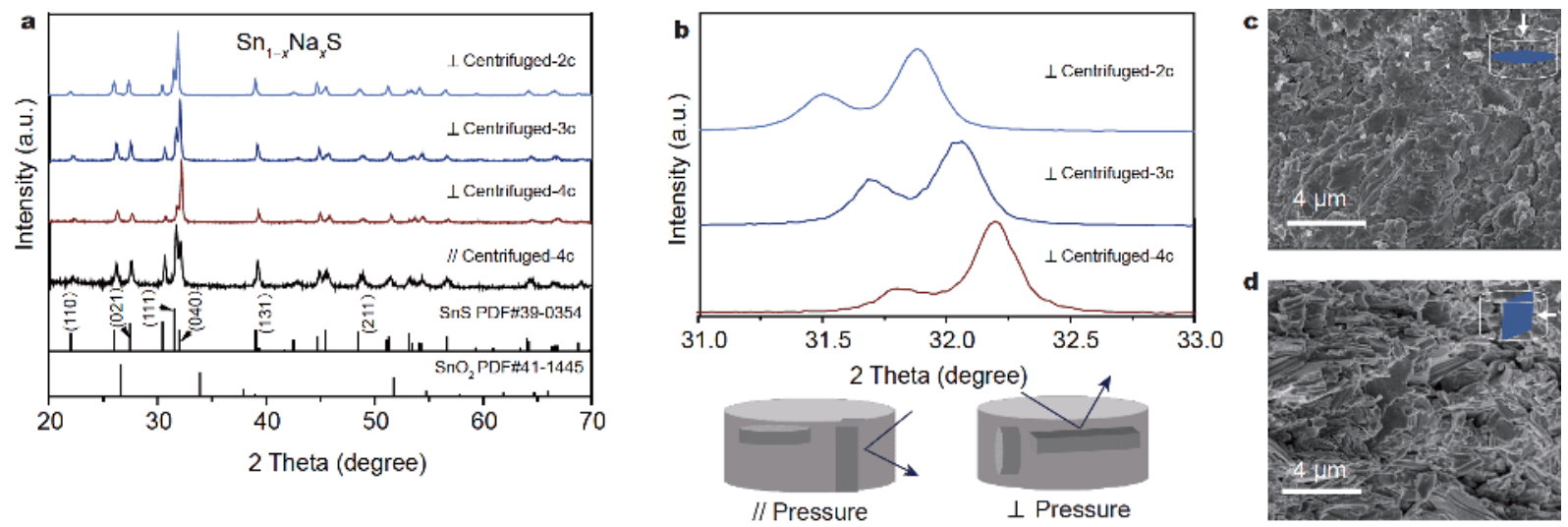

Figure 2 (a) XRD patterns of the samples: Centrifuged-2c, Centrifuged-3c, and Centrifuged-4c bulks perpendicular and parallel to the pressing direction, respectively. The standard low-temperature phase $\mathrm{SnS}$ and $\mathrm{SnO}_{2}$ (PDF\# Nos. 39-0354 and 41-1445). (b) The amplification of the strongest diffraction peak (040). The FESEM images for the representative sample Centrifuged-3c. (c) Image of the bulk perpendicular to the press direction. (d) Image of the bulk parallel to the press direction.

The TE performance varies with direction owing to the non-cubic layered structure and weak van der Waals forces along the $b$-axis. Both the TE properties of $\mathrm{SnS}$ in different directions (i.e., parallel and perpendicular to the pressure axis) were compared and it was found that the performance in the perpendicular direction was slightly higher than that in the parallel direction. Therefore, the samples, Centrifuged- $3 c$ and Centrifuged-2c, were measured in a direction perpendicular to the pressuring direction. Details will be discussed in the following section.

The morphology of the SnS powder was observed via FESEM (Fig. 1c). The SnS powder has two different morphologies, granular and flaky. The size of the granular particles is approximately $100 \mathrm{~nm}$ or even smaller. Interestingly, the flaky SnS are combined into the hollow spheres, which may be closely related to the preparation conditions, thereby affecting the crystal orientation of the sintered SnS bulk.

The XRD patterns of $\mathrm{Sn}_{1-x} \mathrm{Na}_{x} \mathrm{~S}$ bulks are shown in Fig. 2a. Centrifuged-4c, Centrifuged-3c, and Centrifuged$2 c$ represent the samples with corresponding times of centrifugation. Compared with the PDF cards (Nos. 390354 and 41-1445), almost no other phases but SnS appeared. Notably, the ratio of $I_{(040)} / I_{(111)}$ in the perpendicular direction is larger than that in the parallel direction, suggesting that a large number of grains in bulk are oriented. This grain orientation may tend to enhance the electrical transport properties. In addition, with the increasing centrifugation times, the peaks of (111) and (040) gradually shift to the higher angle (Fig. 2b). Based on the Bragg formula, it is easy to understand that the interplanar distance $(d)$ between (111) and (040) is reduced, indicating the influence of centrifugation on the lattice structure of SnS grains. In addition, the orientation relationship of the SnS block was verified by the FESEM images in Fig. $2 \mathrm{c}$ and $\mathrm{d}$. We also found that the centrifugation times, which represent the content of $\mathrm{Na}^{+}$and $\mathrm{Cl}^{-}$ions, have some influence on the morphology of the sintered bulks. As shown in Fig. S2, with the increase in centrifugation times, the lamellar state of the bulks is more evident. The effect of different structures on the TE performance will be exhibited in the following sections.

High-resolution TEM (HRTEM) was performed to observe the detailed crystal morphology, element distribution, and internal defects in the sintered bulks, which may play a significant role on the electrical and thermal properties, as presented in Fig. 3. The Sn and S atoms are expected to be staggered on different columns of the [100] projection (Fig. 3a). The calibrated fast Fourier transformation (FFT), inset in Fig. 3a spots also demonstrate the atomic arrangement. Finally, we did not observe the $\mathrm{Na}^{+}$point defects in the crystal lattice owing to the less amounts of $\mathrm{Na}^{+}$. However, the distribution of $\mathrm{Na}^{+}$in the grains can be clearly observed by the energydispersive X-ray spectrum mapping (Fig. 3b). In addition to the grains with different orientations (Fig. $3 \mathrm{cl}$ and $3 c 2$ ), the orientation relation is not exactly same inside the grain. Abnormally, the long-range ordered structure even inside the grain seems to be interrupted, and the wavy strips in the dark field image (Fig. 3d) clearly prove this phenomenon, which is probably induced by a weak van der Waals force between the Sn-S and Sn-S along the 

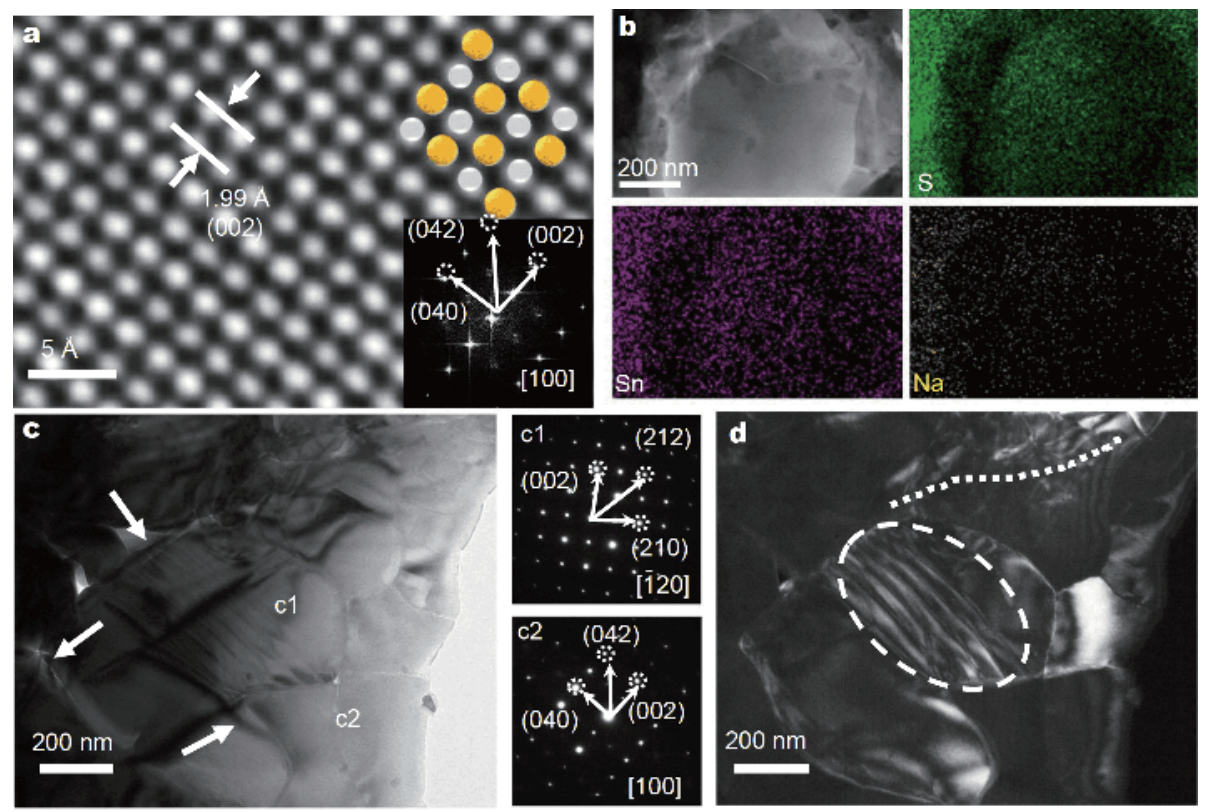

Figure 3 Morphology and structural characterization of the as-prepared Centrifuged-3c sample. (a) HRTEM images along the [100] direction. The insets in (a) are a structure schematic and FFT of the corresponding nanoregion. (b) TEM image and the corresponding elemental mappings: S, Sn, and Na. (c, d) TEM bright field image and dark field image of the bulk. (c1, c2) Corresponding selected area electron diffraction patterns showing the grain phase in the $\mathrm{Sn}_{1-x} \mathrm{Na}_{x} \mathrm{~S}$ bulk.

\section{[010] direction.}

The electrical transport properties for all the samples are illustrated in Fig. 4. The $\sigma$ increases with temperature for the Centrifuged-4c sample. $\sigma$ in the perpendicular direction is slightly greater than that in the parallel direction (Fig. 4a). These results may be caused by the preferred grain orientation in (040) that is confirmed in the XRD pattern. Notably, the two samples are p-type semiconductors with positive $\alpha$ owing to the intrinsic vacancy of $\mathrm{Sn}^{2+}$. Moreover, $\alpha$ in perpendicular direction increases probably because of the strong anisotropy of the effective masses in different crystal directions. Accordingly, PF of Centrifuged-4c in the perpendicular direction has more advantages than that in parallel direction. Therefore, even when high $\sigma$ in the perpendicular direction leads to high thermal conductance $(\kappa)$, the $Z T$ in the perpendicular direction remains high, particularly in high temperature. Thus, the TE properties of Centrifuged- $3 c$ and Centrifuged- $2 c$ were measured in a direction perpendicular to that of the pressure. As shown in Fig. $4 a, \sigma$ of Centrifuged- $3 c$ and Centrifuged $-2 c$ are higher than that of the Centrifuged- $4 c$ sample in the entire temperature range, and the variation rule of $\sigma$ involved three stages, which are consistent with the single ion-doped semiconductors. Generally, because of the higher concentration of $\mathrm{Na}^{+}$ions than that of
Centrifuged-3c, Centrifuged-2c should have higher $\sigma$. However, this phenomenon is not the case at a high temperature stage. The possible reason is that many $\mathrm{Cl}^{-}$ ions have not been completely removed in the matrix when centrifuged only twice. The $\mathrm{Cl}^{-}$not only impedes the migration of carriers and deteriorate the mechanical properties, but also weakens the $\alpha$, as shown in Fig. $4 \mathrm{~b}$. Possibly, the introduction of $\mathrm{Na}^{+}$is always accompanied by impurities. However, when the number of centrifugations is increased to three times, a better balance will exist between the effects of impurities and $\mathrm{Na}^{+}$on the electrical transport performance.

The adjustable amount of doped $\mathrm{Na}^{+}$in the bulk is proven by the Hall carrier concentration $n$ measured at room temperature (Fig. 4c). Evidently, $n$ is negatively correlated with the times of centrifugation. The Hall mobility, $\mu_{\mathrm{H}}$, calculated from $\mu_{\mathrm{H}}=\rho^{-1} e^{-1} n^{-1}$ is simply the opposite. After a certain improvement of $n$, the onset of intrinsic excitations is clearly identified in Fig. S3 on the $n$ of Centrifuged-3c above $700 \mathrm{~K} . \mu_{\mathrm{H}}$ initially rises with the increasing temperature, and then, decreases with regard to the relationship of $\mu_{\mathrm{H}} \approx T^{-1.5}$. This result implies the dominant role of acoustic phonon scattering in the charge transport.

Fig. 4d presents the temperature-dependent $\mathrm{PF}$ of all the SnS samples. The curve of PF for Centrifuged-3c 

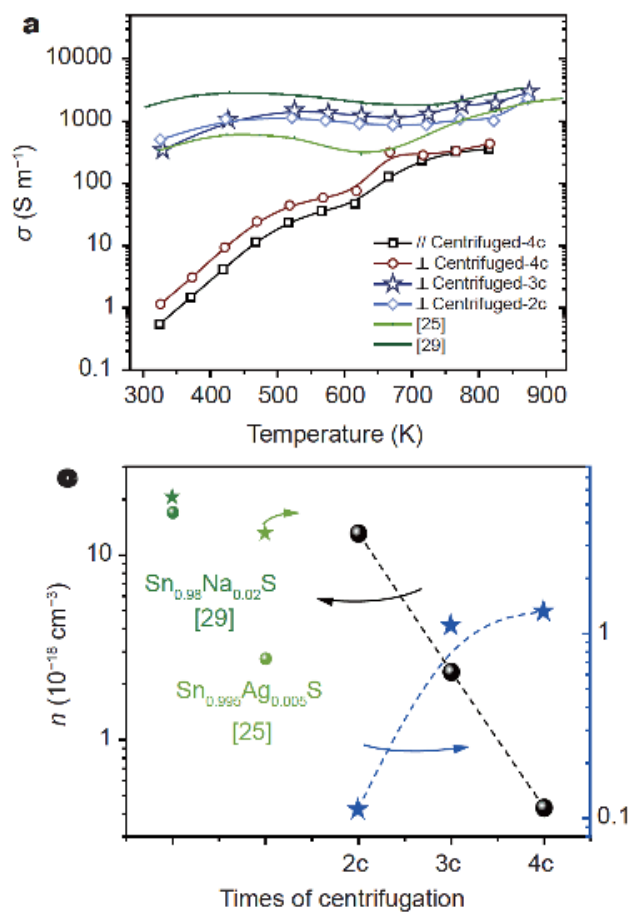
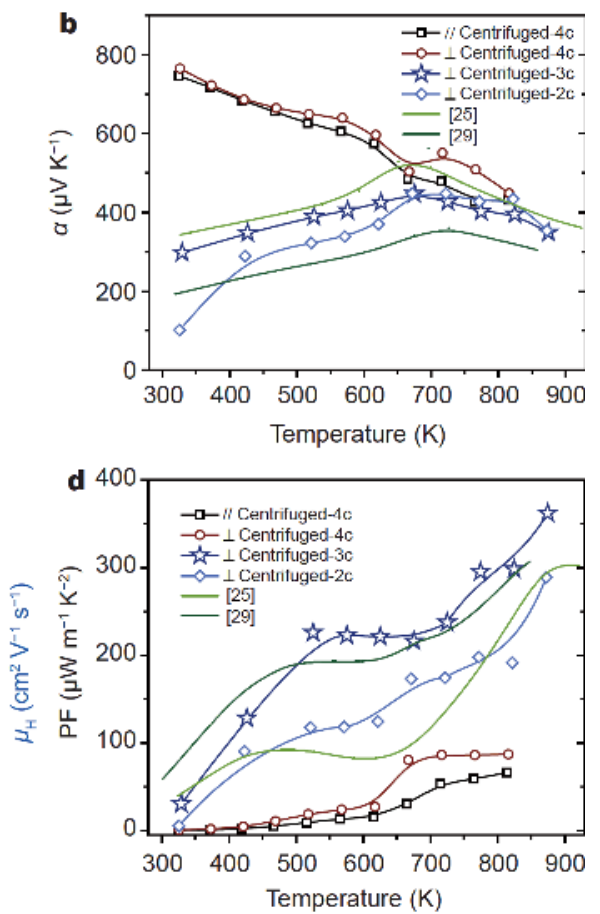

Figure 4 Temperature dependence of electrical properties for the $\mathrm{Sn}_{1-x} \mathrm{Na}_{x} \mathrm{~S}$ bulks when compared with those of $\mathrm{Sn}_{0.95} \mathrm{Ag}_{0.05} \mathrm{~S}$ [25] and $\mathrm{Sn}_{0.98} \mathrm{Na}_{0.02} \mathrm{~S}$ [29]: (a) $\sigma$, (b) $\alpha$, (c) Hall carrier concentration $(n)$ and Hall mobility $\left(\mu_{\mathrm{H}}\right)$ at room temperature, (d) temperature dependence of the PF.

undergoes three stages. This phenomenon is often reported in the doped semiconductor. Finally, we achieved the maximum $\mathrm{PF}$ of $362 \mu \mathrm{W} \mathrm{m}{ }^{-1} \mathrm{~K}^{-2}$ at $873 \mathrm{~K}$ for the sample centrifuged three times, which was substantially higher than that of Centrifuged-4c $\left(<100 \mu \mathrm{W} \mathrm{m}{ }^{-1} \mathrm{~K}^{-2}\right)$ and exceeded the previously reported value for the polycrystalline $\mathrm{SnS}$.

The temperature dependence on the total thermal conductivity, $\kappa_{\text {total }}$, for all the samples is shown in Fig. $5 \mathrm{a}$. For Centrifuged-3c, high $\kappa$ may be attributed to the synergetic effect of the appropriate concentration of $\mathrm{Na}^{+}$, less impurities, better crystallinity, grain orientation, etc. Therefore, we attempted to use a single parabolic bond and Wiedemann-Franz Law model to understand the change on the estimated lattice thermal conductivity $\kappa_{1}=$ $\kappa_{\text {total }}-\kappa_{\mathrm{e}}=\kappa_{\text {total }}-L \sigma T$, where $\kappa_{e}$ and $L$ present the electric thermal conductivity and Lorenz number, respectively. The detailed computational process can be found in the Supplementary information. The temperature-dependent lattice thermal conductivities $\kappa_{1}$ for $\mathrm{Sn}_{1-x} \mathrm{Na}_{x} \mathrm{~S}$ bulks are shown in Fig. 5b. The changing tendency of $\kappa_{1}$ is very similar to that of $\kappa_{\text {total }}$ owing to the low concentration of $\mathrm{Na}$. The theoretical minimum thermal conductivity $\kappa_{\min }$ at $300 \mathrm{~K}$ is shown in Fig. 5b and calculated using Cahill's formula, where the calculated phonon velocities of
$3,368 \mathrm{~m} \mathrm{~s}^{-1}$ for longitudinal and 1,537 and 2,368 $\mathrm{m} \mathrm{s}^{-1}$ for transverse branches at $300 \mathrm{~K}$ are based on relevant literature $[40,41]$. Apparently, $\kappa_{1}$ of all samples each is far higher than the reported values of Ref. 25, Ref. 29, and $\kappa_{\text {min }}$, particularly for the Centrifuged-3c sample. Such a difference may be caused by several reasons. One is that the chemical synthesis can realize the structural reconfiguration at atomic level and achieve amorphous nanoparticles. After the high-temperature SPS sintering, these amorphous nanoparticles can be easily restructured owing to the high-surface-energy. As observed in the XRD patterns and HRTEM images, the grains are arranged with noticeable orientations, which may be more conducive to phonon transmission. Thus, SPS may not be suitable for this case, and alternate technologies, such as cold isostatic pressing combined with rapid thermal processing, should be explored in the future. Meanwhile, given that Cahill's model is based on the assumption that almost all phonons can be scattered in disordered crystals or solid amorphous glass, it is easy to understand the line of $\kappa_{1}$ for all the samples over $\kappa_{\min }$.

Therefore, benefiting from the high PF and weakened bipolar diffusion of $\kappa$, the $Z T$ values for Centrifuged-3c increase rapidly in the entire temperature range, and the largest $Z T$ value reaches 0.52 , which is three times higher 

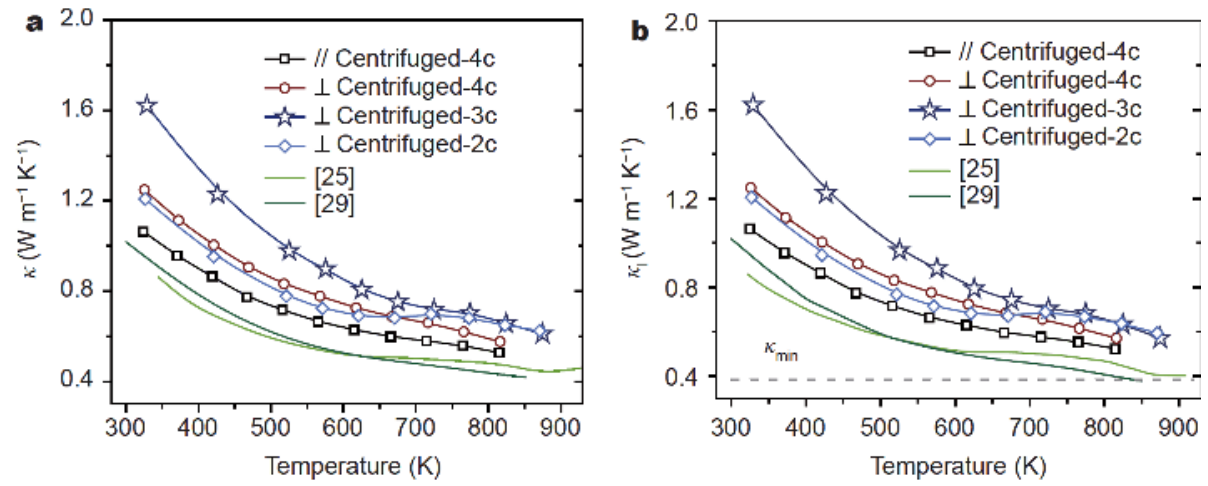

Figure 5 Temperature-dependent total thermal conductivity $\kappa_{\text {total }}$ (a) and lattice thermal conductivity $\kappa_{1}$ (b) for the $\mathrm{Sn}_{1-x} \mathrm{Na}_{x} \mathrm{~S}$ bulks compared with that of the reference value.
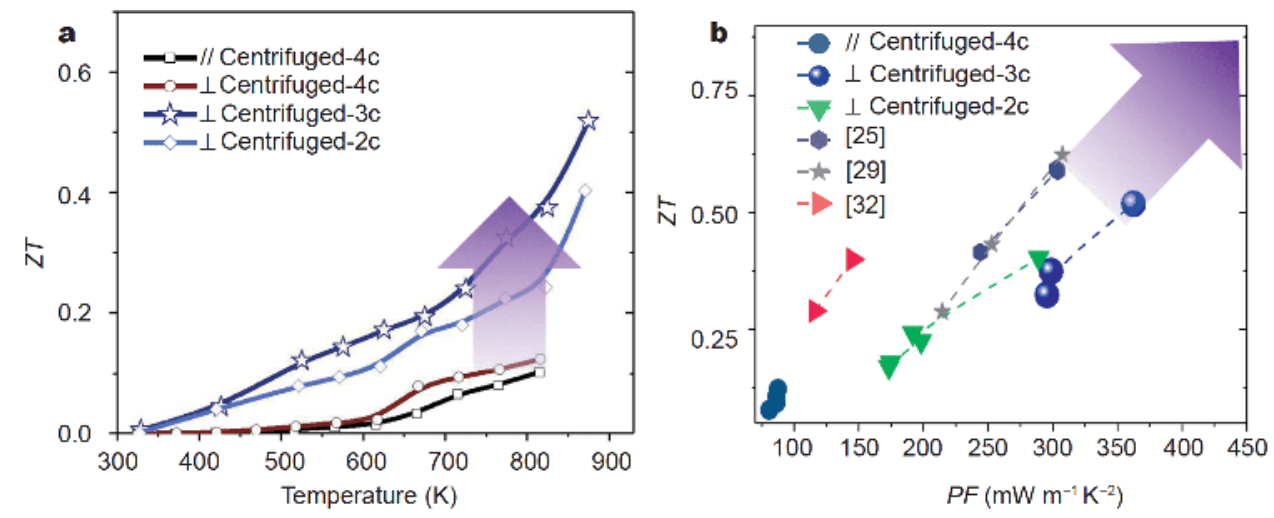

Figure 6 Temperature-dependent $Z T$ values (a) and $Z T$ vs. PF plot (b) for the $\mathrm{Sn}_{1-x} \mathrm{Na}_{x} \mathrm{~S}$ bulks compared with that of the reference data.

than that of the reference SnS (Fig. 6a). Moreover, further $Z T$ enhancement is highly possible by employing nanostructuring approaches to reduce thermal conductivity.

\section{CONCLUSION}

In summary, we synthesized the polycrystalline $\mathrm{Na}$-doped SnS with enhanced TE performance using the improved chemical co-precipitation. Different filtration conditions were explored to adjust the content of doped $\mathrm{Na}^{+}$ion in SnS bulks. The optimized condition leads to a maximum $\mathrm{PF}$ of up to $362 \mu \mathrm{W} \mathrm{m} \mathrm{m}^{-1} \mathrm{~K}^{-2}$ at $873 \mathrm{~K}$, which is the highest reported value for the polycrystalline SnS. Even without intentional chemical doping, the present SnS materials have high $Z T$ up to 0.52 at $873 \mathrm{~K}$, despite its higher thermal conductivity. This study offers an effective approach and perspective to significantly improve $\mathrm{PF}$ and achieve high $Z T$ in SnS. Therefore, besides $\mathrm{Na}$ doping, we highly expect that this modification process can be extended to other dopants that have similar cation doping efficiency and can broaden the scope of compound technology.

Received 2 December 2018; accepted 3 January 2019;

published online 28 January 2019

1 Bonaccorso F, Colombo L, Yu G, et al. Graphene, related twodimensional crystals, and hybrid systems for energy conversion and storage. Science, 2015, 347: 1246501

2 Zhao LD, Lo SH, Zhang Y, et al. Ultralow thermal conductivity and high thermoelectric figure of merit in SnSe crystals. Nature, 2014, 508: 373-377

3 Duong AT, Nguyen VQ, Duvjir G, et al. Achieving $Z T=2.2$ with Bi-doped n-type SnSe single crystals. Nat Commun, 2016, 7: 13713

4 Wan C, Gu X, Dang F, et al. Flexible n-type thermoelectric materials by organic intercalation of layered transition metal dichalcogenide $\mathrm{TiS}_{2}$. Nat Mater, 2015, 14: 622-627

5 Zhang K, Davis M, Qiu J, et al. Thermoelectric properties of porous multi-walled carbon nanotube/polyaniline core/shell nanocomposites. Nanotechnology, 2012, 23: 385701

6 Juang ZY, Tseng CC, Shi Y, et al. Graphene-Au nanoparticle based vertical heterostructures: A novel route towards high-ZT thermoelectric devices. Nano Energy, 2017, 38: 385-391

7 Deng Z, Han D, Liu Y. Colloidal synthesis of metastable zinc- 
blende IV-VI SnS nanocrystals with tunable sizes. Nanoscale, 2011, 3: 4346-4351

8 Ge ZH, Zhang BP, Chen YX, et al. Synthesis and transport property of $\mathrm{Cu}_{1.8} \mathrm{~S}$ as a promising thermoelectric compound. Chem Commun, 2011, 47: 12697-12699

9 Fan DD, Liu HJ, Cheng L, et al. $\mathrm{MoS}_{2}$ nanoribbons as promising thermoelectric materials. Appl Phys Lett, 2014, 105: 133113

10 Lin Y, Norman C, Srivastava D, et al. Thermoelectric power generation from lanthanum strontium titanium oxide at room temperature through the addition of graphene. ACS Appl Mater Interfaces, 2015, 7: 15898-15908

11 Lou XW, Yuan C, Archer LA. Shell-by-shell synthesis of tin oxide hollow colloids with nanoarchitectured walls: cavity size tuning and functionalization. Small, 2007, 3: 261-265

12 Dey A, Bajpai OP, Sikder AK, et al. Recent advances in CNT/ graphene based thermoelectric polymer nanocomposite: A proficient move towards waste energy harvesting. Renew Sustain Energy Rev, 2016, 53: 653-671

13 Slack GA. Design concepts for improved thermoelectric materials. MRS Online Proceedings Library Archive. 1997, 478

14 Tritt TM. Holey and unholey semiconductors. Science, 1999, 283: 804-805

15 Min G, Rowe DM. A serious limitation to the phonon glass electron crystal (PGEC) approach to improved thermoelectric materials. J Mater Sci Lett, 1999, 18: 1305-1306

16 Li J, Zhang X, Chen Z, et al. Low-symmetry rhombohedral GeTe thermoelectrics. Joule, 2018, 2: 976-987

17 Tang Y, Gibbs ZM, Agapito LA, et al. Convergence of multi-valley bands as the electronic origin of high thermoelectric performance in $\mathrm{CoSb}_{3}$ skutterudites. Nat Mater, 2015, 14: 1223-1228

18 Chen Z, Ge B, Li W, et al. Vacancy-induced dislocations within grains for high-performance PbSe thermoelectrics. Nat Commun, 2017, 8: 13828

19 Chen Z, Zhang X, Lin S, et al. Rationalizing phonon dispersion for lattice thermal conductivity of solids. Natl Sci Rev, 2018, 5: 888894

20 Hong $\mathrm{M}$, Chen ZG, Yang $\mathrm{L}$, et al. $\mathrm{Bi}_{x} \mathrm{Sb}_{2-x} \mathrm{Te}_{3}$ nanoplates with enhanced thermoelectric performance due to sufficiently decoupled electronic transport properties and strong wide-frequency phonon scatterings. Nano Energy, 2016, 20: 144-155

21 Poudel B, Hao Q, Ma Y, et al. High-thermoelectric performance of nanostructured bismuth antimony telluride bulk alloys. Science, 2008, 320: 634-638

22 Dong J, Liu W, Li H, et al. In situ synthesis and thermoelectric properties of $\mathrm{PbTe}$-graphene nanocomposites by utilizing a facile and novel wet chemical method. J Mater Chem A, 2013, 1: 12503

23 Jiang G, He J, Zhu T, et al. High performance $\mathrm{Mg}_{2}(\mathrm{Si}, \mathrm{Sn})$ solid solutions: a point defect chemistry approach to enhancing thermoelectric properties. Adv Funct Mater, 2014, 24: 3776-3781

24 Jiang B, Qiu P, Chen $\mathrm{H}$, et al. An argyrodite-type $\mathrm{Ag}_{9} \mathrm{GaSe}_{6}$ liquidlike material with ultralow thermal conductivity and high thermoelectric performance. Chem Commun, 2017, 53: 11658-11661

25 Li F, Li JF, Zhao LD, et al. Polycrystalline $\mathrm{BiCuSeO}$ oxide as a potential thermoelectric material. Energy Environ Sci, 2012, 5: 7188

$26 \mathrm{Wu} \mathrm{H}$, Lu X, Wang G, et al. Sodium-doped tin sulfide single crystal: A nontoxic earth-abundant material with high thermoelectric performance. Adv Energy Mater, 2018, 8: 1800087
27 Sun FH, Dong J, Dey S, et al. Enhanced thermoelectric performance of $\mathrm{Cu}_{12} \mathrm{Sb}_{4} \mathrm{~S}_{13-\delta}$ tetrahedrite via nickel doping. Sci China Mater, 2018, 61: 1209-1217

28 He W, Wang D, Dong JF, et al. Remarkable electron and phonon band structures lead to a high thermoelectric performance $Z T>1$ in earth-abundant and eco-friendly SnS crystals. J Mater Chem A, 2018, 6: 10048-10056

29 Zhou B, Li S, Li W, et al. Thermoelectric properties of SnS with Na-doping. ACS Appl Mater Interfaces, 2017, 9: 34033-34041

30 Tang H, Sun FH, Dong JF, et al. Graphene network in copper sulfide leading to enhanced thermoelectric properties and thermal stability. Nano Energy, 2018, 49: 267-273

31 Wan $\mathrm{C}$, Wang $\mathrm{Y}$, Wang $\mathrm{N}$, et al. Low-thermal-conductivity $(\mathrm{MS})_{1+x}\left(\mathrm{TiS}_{2}\right)_{2}(\mathrm{M}=\mathrm{Pb}, \mathrm{Bi}, \mathrm{Sn})$ misfit layer compounds for bulk thermoelectric materials. Materials, 2010, 3: 2606-2617

32 Wei TR, Qin Y, Deng T, et al. Copper chalcogenide thermoelectric materials. Sci China Mater, 2019, 62: 8-24

33 Tan Q, Zhao LD, Li JF, et al. Thermoelectrics with earth abundant elements: low thermal conductivity and high thermopower in doped SnS. J Mater Chem A, 2014, 2: 17302-17306

34 Tan Q, Li JF. Thermoelectric properties of Sn-S bulk materials prepared by mechanical alloying and spark plasma sintering. J Elec Materi, 2014, 43: 2435-2439

35 Peng K, Lu X, Zhan H, et al. Broad temperature plateau for high ZTs in heavily doped p-type SnSe single crystals. Energy Environ Sci, 2016, 9: 454-460

36 Wang C, Chen Y, Jiang J, et al. Improved thermoelectric properties of SnS synthesized by chemical precipitation. RSC Adv, 2017, 7: $16795-16800$

37 Sun BZ, Ma Z, He C, et al. Anisotropic thermoelectric properties of layered compounds in $\mathrm{SnX}_{2}(\mathrm{X}=\mathrm{S}, \mathrm{Se})$ : a promising thermoelectric material. Phys Chem Chem Phys, 2015, 17: 29844-29853

38 Zhang Q, Chere EK, Sun J, et al. Studies on thermoelectric properties of $\mathrm{n}$-type polycrystalline $\mathrm{SnSe}_{1-x} \mathrm{~S}_{x}$ by iodine doping. Adv Energy Mater, 2015, 5: 1500360

39 Herron SM, Tanskanen JT, Roelofs KE, et al. Highly textured tin (II) sulfide thin films formed from sheetlike nanocrystal inks. Chem Mater, 2014, 26: 7106-7113

40 Cahill DG, Watson SK, Pohl RO. Lower limit to the thermal conductivity of disordered crystals. Phys Rev B, 1992, 46: 61316140

41 Ding G, Gao G, Yao K. High-efficient thermoelectric materials: The case of orthorhombic IV-VI compounds. Sci Rep, 2015, 5: 9567

Acknowledgements This work was supported by the National Key R\&D Program of China (2018YFB0703603), the Basic Science Center Project of National Natural Science Foundation of China (NSFC, 51788104), and the NSFC (11474176).

Author contributions Tang $\mathrm{H}$ and $\mathrm{Li} \mathrm{JF}$ designed the project; Tang $\mathrm{H}$, Dong JF, Sun FH, Asfandiyar, and Shang P conducted experiments and analyzed the data; Tang $\mathrm{H}$ wrote the paper with support from Li JF. All authors contributed to the general discussion.

Conflict of interest The authors declare no conflict of interest

Supplementary information Supporting data are available in the online version. 


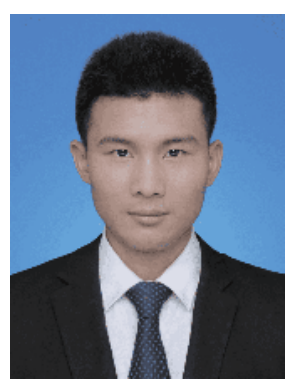

Huaichao Tang is a PhD candidate in the School of Materials Science and Engineering, Tsinghua University. His current research focuses on the synthesis of chalcogenides and their applications in thermoelectric.

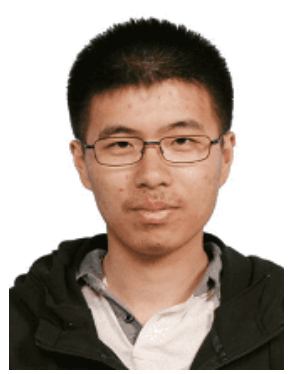

Jin-Feng Dong is a PhD candidate in the School of Materials Science and Engineering, Tsinghua University. His current research focuses on the synthesis of manganese-based materials and their applications in thermoelectric.

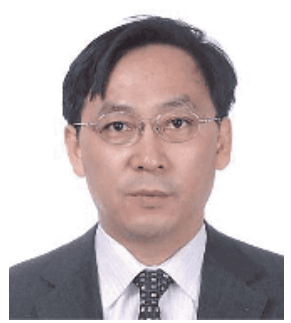

Jing-Feng Li received PhD from Tohoku University in 1991, and worked there as an assistant professor and associate professor until joining Tsinghua University as a full professor in 2002. He is currently Cheung Kong Scholar Distinguished Professor at Tsinghua University. His research interest includes piezoelectric and thermoelectric materials and MEMS technologies. He is the Editor-in-Chief of Journal of Materiomics.

\section{湿化学方法掺杂Na对多晶SnS热电性能的影响}

唐怀超 ${ }^{1}$, 董金峰 ${ }^{1}$, 孙富华 ${ }^{1}$, Asfandiyar ${ }^{1}$, 尚鹏鹏 ${ }^{2}$, 李敬锋 ${ }^{*}$

摘要 $\mathrm{SnS}$ 作为一种与 $\mathrm{SnSe}$ 特征结构相似且元素丰度更高的热电材料, 受到越来越多的关注, 但是其较低的本征载流子浓度限制了热电性 能的提升. 本工作利用了一种改进的化学共沉淀方法调节基体中 $\mathrm{Na}^{+}$含量来提高载流子浓度, 进而提升了多晶 $\mathrm{SnS}$ 的热电性能. 最大功率因 子在 $873 \mathrm{~K}$ 达到 $362 \mu \mathrm{W} \mathrm{m} \mathrm{m}^{-1} \mathrm{~K}^{-2}$, 高于目前关于多晶 $\mathrm{SnS}$ 的最高报道值. 得益于提升的电输运性能以及较低的热导率, $Z T$ 值在 $873 \mathrm{~K}$ 达到 0.52. 该工作为其他热电化合物的掺杂改性技术提供了新思路. 